\title{
Hypercholesterolemia boosts joint destruction in chronic arthritis. An experimental model aggravated by foam macrophage infiltration
}

\author{
I Prieto-Potín, JA Roman-Blas, MJ Martínez-Calatrava, R Gómez, R Largo and Gabriel Herrero-Beaumont
}

\begin{abstract}
Objective: The aim of this study was to determine whether hypercholesterolemia increases articular damage in a rabbit model of chronic arthritis.

Methods: Hypercholesterolemia was induced in 18 rabbits by administrating a high-fat diet (HFD). Fifteen rabbits were fed normal chow as controls. Chronic antigen-induced arthritis (AIA) was induced in half of the HFD and control rabbits, previously immunized, by intra-articular injections of ovalbumin. After sacrifice, lipid and systemic inflammation markers were analyzed in blood serum. Synovium was analyzed by Krenn score, multinucleated cell counting, immunohistochemistry of RAM11 and CD31, and TNF- $\alpha$ and macrophage chemoattractant protein-1 (MCP-1) gene expression. Active bone resorption was assessed by protein expression of receptor activator of nuclear factor kappa-B ligand (RANKL), osteoprotegerin (OPG) and quantification of cathepsin K, contact surface and the invasive area of pannus into bone.

Results: Rabbits receiving the HFD showed higher total serum cholesterol, HDL, triglycerides and CRP levels than rabbits fed a normal diet. Synovitis score was increased in HFD, and particularly in AIA and AIA + HFD groups. AIA + HFD synovium was characterized by a massive infiltration of RAM11+ cells, higher presence of multinucleated foam cells and bigger vascularization than AIA. Cathepsin K+ osteoclasts and the contact surface of bone resorbing pannus were also increased in rabbits with AIA + HFD compared with AIA alone. Synovial TNF- $\alpha$ and MCP-1 gene expression was increased in AIA and HFD rabbits compared with healthy animals. RANKL protein expression in AIA and AIA + HFD groups was higher compared with either HFD or normal groups.
\end{abstract}

Conclusions: This experimental model demonstrates that hypercholesterolemia increments joint tissue damage in chronic arthritis, with foam macrophages being key players in this process.

\section{Introduction}

The increased burden of cardiovascular disease in rheumatoid arthritis (RA) is only explained partly by traditional cardiovascular risk factors. They seem to have similar prevalence in RA and non-RA patients, suggesting that other factors contribute significantly to this health issue [1,2]. In fact, non-traditional risk factors, such as RA disease activity/severity measures and some antirheumatic drugs, have been consistently associated with increased cardiovascular risk. Thus, RA inflammation

\footnotetext{
* Correspondence: gherrero@fjd.es

Bone and Joint Research Unit, Service of Rheumatology, IIS Fundación Jiménez Díaz, Universidad Autónoma, Av. Reyes Católicos 2, 28048, Madrid,
} Spain

(c) 2013 Prieto-Potín et al.; licensee BioMed Central Ltd. This is an Open Access article distributed under the terms of the Creative Commons Attribution License (http://creativecommons.org/licenses/by/2.0), which permits unrestricted use, distribution, and reproduction in any medium, provided the original work is properly cited. plays a notable role in the development of cardiovascular disease.

Chronic synovitis is a source of inflammatory mediators that include cytokines, chemokines and adipokines, such as TNF- $\alpha$, macrophage chemoattractant protein-1 (MCP-1), plasminogen activator inhibitor-1, interleukin (IL) -6 and others. In addition to the production of most of these pro-inflammatory cytokines, there is increasing evidence concerning the contribution of dysregulated adipose tissue through adipokine secretion to systemic RA inflammation [3]. Indeed, the inflammatory actions exerted by adipokines could explain some of the association between several rheumatic diseases and cardiovascular comorbidities [4]. A relevant role has been suggested for leptin in immunity, not only by maintaining energy 
homeostasis but also by regulating the function of immune cells. Specifically, leptin has been shown to promote phagocytic function and induce production of several pro-inflammatory cytokines in macrophages and monocytes [5]. Serum resistin levels have been shown to be higher in patients with RA than in healthy controls and correlate with inflammation and joint damage. In RA, macrophages, among other immune cells, showed co-localization with resistin [6]. An increase of leptin and resistin (pro-atherogenic hormones) and the decrease of adiponectin (anti-atherogenic hormone) may alter endothelial homeostasis in RA patients [7].

Endothelial dysfunction occurs in early stages of RA and atherosclerosis as a response to the raised expression of chemokines and adhesion molecules, promoting the enhancement of vessel wall permeability. These events favor leukocyte trafficking toward the site of synovial inflammation in arthritis [8] and/or promote infiltration of lipids, monocytes and T-lymphocytes. Further appearance of foam cells and fatty streaks within the vessel wall originates the formation of atherosclerotic plaques [9]. In addition, recent data showed that the vascular regenerative action of endothelial progenitor cells is altered in RA and atherosclerosis [10-13]. Therefore, the increase of pro-inflammatory signals and the impairment of reparatory processes, both mechanisms shared between RA and atherosclerosis, may contribute significantly to joint damage in RA.

The role of hypercholesterolemia in the progression of atherosclerosis is well established [14,15]. Severe hypercholesterolemia aggravated atherosclerotic plaques instability and aortic lesions mainly due to massive $\mathrm{M} \phi$ infiltration in our animal rabbit model of atherosclerosis associated with chronic arthritis [16]. Macrophages $(\mathrm{M} \phi)$ act as immune innate cells, antigen presenting cells and, finally, effector cells for joint inflammation in both acute and chronic phases. Indeed, $\mathrm{M} \phi$ contribute to the hyperplasia of the lining layer, and are the main cells in the mononuclear infiltration of the synovial sublining and the cartilage-pannus junction, as well as, the major producers of prominent inflammatory mediators. Activated $\mathrm{M} \phi$ may also differentiate into osteoclast-like cells and become involved in bone resorption [17-20]. Because relevant pathophysiological mechanisms occur similarly in both atherosclerosis and chronic inflammatory states, we hypothesize that hypercholesterolemia may also significantly contribute to joint damage in chronic arthritis through the enhancement of $\mathrm{M} \phi$ aggressiveness, as suggested in early findings in our previous study [16].

In this regard, significant research efforts have just begun to explore the mechanisms underlying the influence of hypercholesterolemia in the development and progression of inflammatory arthritis. During hyperlipidemia, adipocytes release well-known pro-inflammatory mediators and adipokines that play relevant roles in inflammatory arthritides [21]. A lipid-rich diet has been shown to contribute to the switch in polarization of adipose tissue $\mathrm{M} \phi$ from an anti-inflammatory (M2) to a pro-inflammatory (M1) state associated with obesityinduced insulin resistance [22]. This change induces systemic inflammation and potentially increases the progression of chronic arthritis. Hyperlipidemia has also been suggested to promote the osteoclastic potential of bone marrow cells in vivo. Indeed, the presence of lipid oxidation products in bone marrow and an increased osteoclast size in bones indicates a functional, but not numeric, difference in osteoclasts as described in hyperlipidemic mice [23]. Furthermore, diet-induced hypercholesterolemia in mice was associated with reduced bone quality measures that resemble human bone with osteoporosis [24]. Thus, hypercholesterolemia may start a systematic loss of bone homeostasis.

Therefore, in this study we determine whether hypercholesterolemia increases articular damage in a rabbit model of chronic arthritis and if this effect occurs through the activation of the synovial mononuclear phagocyte system.

\section{Material and methods}

\section{Experimental model in rabbits}

Adult male New Zealand rabbits with a body weight of 3 to $3.5 \mathrm{~kg}$ (Granja San Bernardo, Navarra, Spain) were used for the experimental procedures. Animal handling and experimentation were performed in accordance with Spanish Regulations and the Guidelines for the Care and Use of Laboratory Animals drawn up by the National Institutes of Health (Bethesda, MD, USA). The experimental protocol was approved by the Institutional Ethics Committee.

After two weeks of adaptation to our facilities, 18 rabbits were fed ad libitum with a high-fat diet (HFD) consisting of $1 \%$ cholesterol $+3 \%$ peanut oil (Harlan, Inc., Indianapolis, IN, USA). One week later, antigen-induced arthritis (AIA) was induced in half of these animals $(n=9$; AIA + HFD) according to a protocol previously described [16]. Briefly, animals were given two intradermal injections of $1 \mathrm{ml}$ ovalbumin (OVA) $(4 \mathrm{mg} / \mathrm{ml}$; Sigma-Aldrich, St. Louis, MO, USA) in Freund's complete adjuvant (Difco, Detroit, MI, USA). Five days after the second injection, $1 \mathrm{ml}$ of OVA $(5 \mathrm{mg} / \mathrm{ml}$ in $0.9 \% \mathrm{NaCl})$ was injected intraarticularly into the knee joint on a weekly basis over the following four weeks (Figure 1A). The other half of HFDfed rabbits underwent no injections $(n=9$; HFD). We simultaneously induced AIA in a group of nine rabbits receiving standard chow. From this group, two rabbits died for unknown reasons and the remaining rabbits went through the whole study ( $n=7$; AIA). Eight additional rabbits fed with standard chow and spared from any experimental intervention were used as healthy controls. 


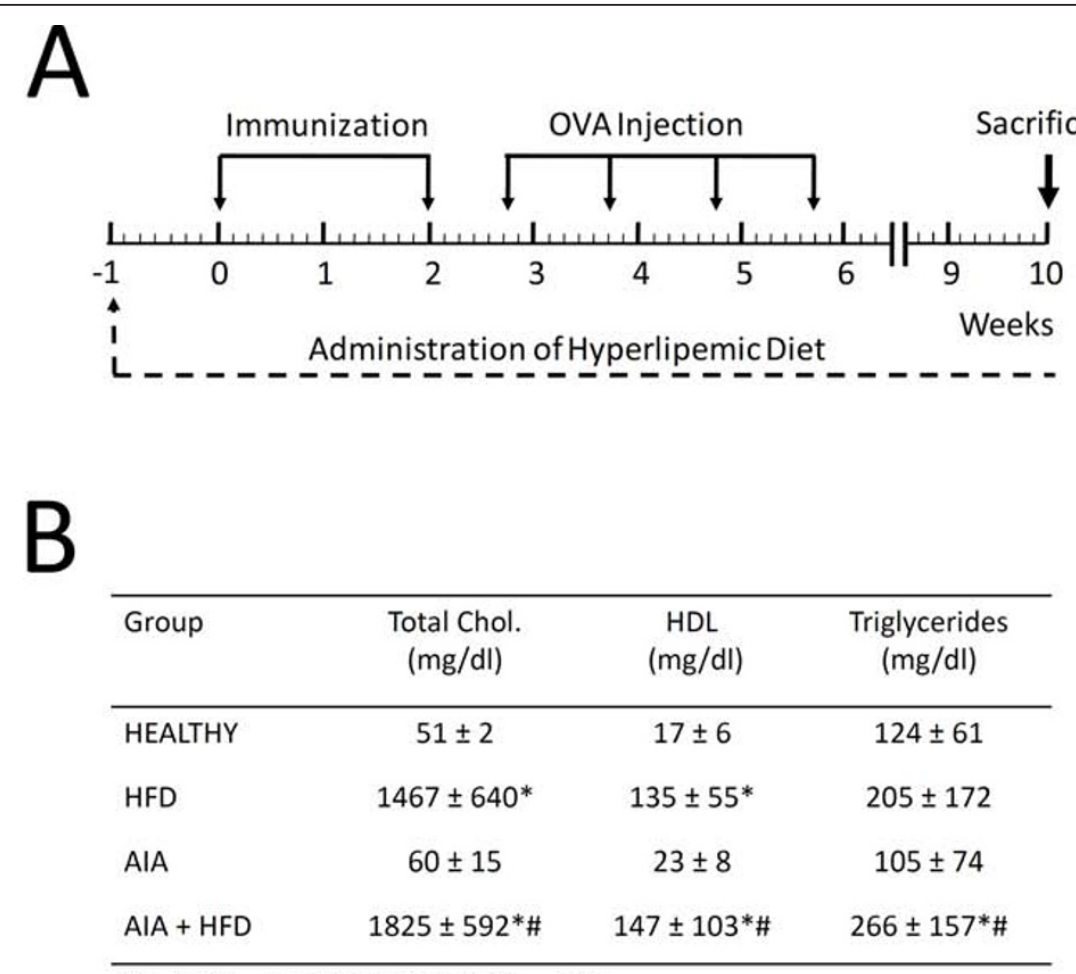

$* P<0,01$ vs HEALTHY; \# $P<0,01$ vs AIA
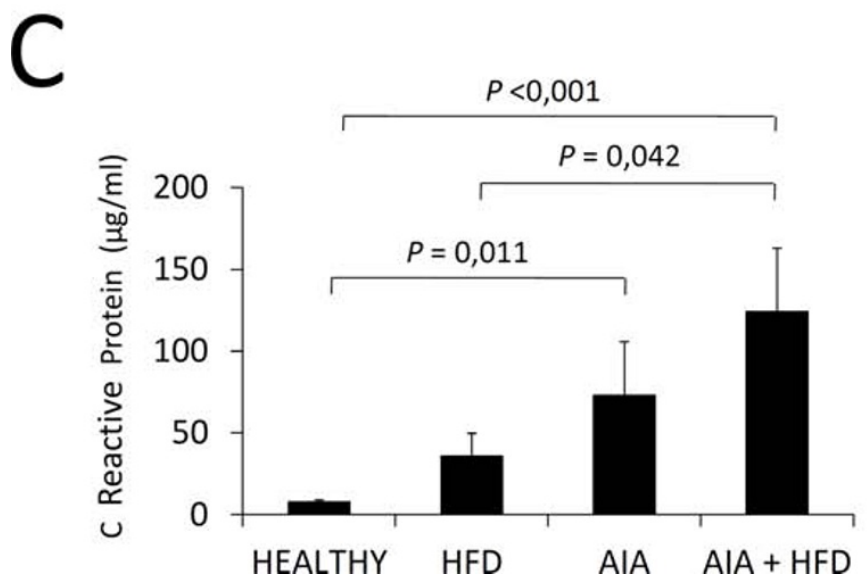

Figure 1 Effect of the hyperlipemic diet and chronic antigen-induced arthritis (AIA) on serum content. A, Schematic representation of the experimental model. B, Total cholesterol (Total Chol), high density lipoprotein (HDL) cholesterol and triglycerides levels $(\mathrm{mg} / \mathrm{dl})$ in the sera of healthy rabbits, high-fat diet (HFD) rabbits, AIA rabbits and AIA + HFD rabbits. C, Concentration of C reactive protein $(\mu \mathrm{g} / \mathrm{ml})$ in the sera of experimental rabbits. Bars show the mean and SEM ( $n=7$ to 9 rabbits per group). OVA, Ovalbumin.

At the end of the study, rabbits were bled from their marginal ear vein, and then euthanized with an overdose of pentobarbital (Braun Medical SA, Barcelona, Spain) in order to evaluate chronic damage. The synovial membranes of the right knee of each rabbit were fixed in $4 \%$ buffered paraformaldehyde and embedded in paraffin for histological studies to be performed. The synovial membranes from the left knees were immediately frozen and used for molecular biology studies. Both femurs were fixed, decalcified for four weeks in $10 \%$ formic acid plus $5 \%$ paraformaldehyde, and then embedded in paraffin. Articular cartilage and subchondral bone were obtained from both tibias for gene and protein expression studies.

\section{Serum chemistry}

Ten milliliters of blood were used for serum extraction. Total and high-density lipoprotein (HDL) cholesterol and triglycerides were measured by Advia ${ }^{\circledR} 2400$ 
chemistry system (Siemens Healthcare Diagnostics, Tarrytown, NY, USA). A specific enzyme-linked immunosorbent assay kit was used to measure C-reactive protein (CRP; Alpha Diagnostic, San Antonio, TX, USA).

\section{Histopathology}

Synovial histopathology was evaluated in hematoxylineosin (H-E) stained sections by two blinded observers, according to the Krenn scale, as previously described [25]. Briefly, lining hyperplasia, fibrovascular alterations at the interstitium, and the tissue infiltration were independently evaluated using 0 to 3 -point subscales, where 0 indicates absence, 1 mild, 2 intermediate and 3 strong. The total score was obtained from the sum of partial grades with a maximum total score of 9 [26].

Multinucleated cell counting was performed in $\mathrm{H}-\mathrm{E}$ sections by direct analysis of 10 randomly chosen microscopic fields, where lining and sublining layers were identifiable by the observer at 20x magnification.

In order to evaluate the invasiveness of the synovium at the posterior bone-pannus interface region, we quantified the extent of the contact surface between the pannus and bone/calcified cartilage, and the area of invasive pannus in contact with bone in H-E sections of femoral condyles. The microphotographs of the posterior bone-pannus interface region at $40 \mathrm{x}$ magnification of each rabbit were analysed. All samples were positioned in the same plane in order to assess a similar area in each measurement. For contact surface, the length of the boundary line between the pannus and bone/calcified cartilage tissue was measured. For the quantification of the invasive area, each tissue (bone, synovial membrane or cartilage) was identified by a distinctive color and then the area of each color was measured. The number of cathepsin K-positive osteoclasts was assessed in the same localization in order to estimate active bone resorption, and was expressed as positive cells per area. The assessment of these parameters was carried out with Image-Pro Plus software (version 4.5 for Windows, Media Cybernetics, Inc, Silver Spring, MD, USA).

\section{Immunohistochemistry}

In the synovial membrane, we identified macrophages using a monoclonal anti-rabbit macrophage antibody (RAM11; Dako, Glostrup, Denmark), according to protocol [27]. The antibody was detected with a biotinylated goat anti-mouse IgG (1:200; Amersham, Arlington Heights, IL, USA) visualized with a horseradish peroxidase/ABComplex using 3,3diaminobenzidine tetrahydrochloride as the chromogen (Dako, Camarillo, CA, USA). The tissues were counterstained with hematoxylin and mounted in DPX medium (VWR International, Leuven, Belgium). Computer-assisted analysis was performed with Leica DMD108 Digital Micro Imaging device (Leica,
Microsystems, Inc., Buffalo Grove, IL, USA) and with Image-Pro Plus software (version 4.5 for Windows, Media Cybernetics, Inc, Silver Spring, MD, USA)). The results were expressed as percentage of positive area. An IgG isotype was used as a negative control.

CD31 immunostaining (Abcam, Cambridge, UK) was assessed in the synovial membrane, as a marker of vascular endothelial cells. Briefly, synovium sample slides were scanned in the Coreo Iscan Au scanner (Ventana Medical Systems, Tucson, AZ, USA) and then total cells, CD31positive cells and the entire sample area were assessed with Virtuoso Image management software (Ventana Medical Systems, Tucson, AZ, USA). The results were expressed as the ratio between CD31positive cells and the area in $\mathrm{mm}^{2}$.

Cathepsin K-positive cells were evaluated in femur sections in order to assess bone resorption (Abcam, Cambridge, UK). Briefly, the number of cathepsin K-positive multinucleated cells was assessed at the bone-pannus interface region of AIA and AIA + HFD knees. All samples were positioned in the same plane in order to assess a similar area in each measurement, and the results are shown as positive staining per area.

\section{Gene expression analysis}

Total RNA was isolated from synovial membranes using TriPure Isolation Reagent (Roche Diagnostics, Indianapolis, IN, USA), according to the manufacturer's instructions. A total of $1 \mu \mathrm{g}$ RNA was reverse-transcribed with the high capacity cDNA kit (Applied Biosystems, Foster City, CA, USA) and RNA expression was quantified by single-reporter real time PCR using the StepOnePlus ${ }^{\mathrm{TM}}$ detection system and StepOne ${ }^{\mathrm{TM}}$ software v2.2 (Applied Biosystems). Specific oligonucleotide fluorescently labeled primers, TaqMan FAM probe assay for TNF- $\alpha$ (Oc 03397715_m1) and assay-on-demand for MCP-1 were purchased from Applied Biosystems. A pre-designed TaqMan FAM probe assay for GAPDH (Oc 03823402_g1, Applied Biosystems) was also used as an endogenous control, and mRNA expression was normalized to GAPDH RNA in each well.

\section{Western blot analysis}

Tissues were homogenized in liquid nitrogen, and total proteins were extracted employing an extraction buffer containing 15 mM HEPES, 10\% glycerol, 0.5\% NP-40, $250 \mathrm{mM} \mathrm{NaCl}, 1 \mathrm{mM}$ EDTA, 1:1,000 phenylmethanesulfonylfluoride (PMSF) and a protease inhibitor cocktail (Sigma-Aldrich). Protein concentration was determined as previously described [28], and subsequently, $20 \mu \mathrm{g}$ of total protein from each tissue was resolved on 15\% acrylamideSDS gels. After transfer to polyvinylidene difluoride (PVDF) membranes (Millipore, Molsheim, France) in $48 \mathrm{mM}$ Tris, $39 \mathrm{mM}$ glycine and 20\% methanol buffer at 
$20 \mathrm{~V}$ for $1 \mathrm{~h}$ at room temperature, membranes were blocked in 5\% skimmed milk in PBS-Tween 20 for $1 \mathrm{~h}$ at room temperature and incubated overnight at $4^{\circ} \mathrm{C}$ with anti-receptor activator of nuclear factor kappa-B ligand (RANKL) antibodies (Peprotech, Neuilly-Sur-Seine, France) and anti-osteoprotegerin (OPG) (R\&D Systems, Minneapolis, MN, USA) at 1/1,000 dilution each. Antibody binding was detected by enhanced chemoluminescence using peroxidase-labeled secondary antibodies, and the results were expressed as arbitrary densitometric units (AU). Loading control was performed on $15 \%$ acrylamideSDS gels by employing EZBlue gel staining reagent (Sigma-Aldrich).

\section{Statistical analysis}

All statistical analyses were performed using SPSS version 17.0 software for Windows (SPSS, Chicago, IL, USA), and results were expressed as the mean \pm standard error of mean (SEM). The data from multiple groups were compared using a Kruskal-Wallis nonparametric test, and a pairwise comparison using the Mann-Whitney test was applied when overall differences were identified. $P$-values $<0.05$ were considered significant.

\section{Results}

\section{Metabolic profile}

Rabbits fed with a hyperlipemic diet (the HFD and AIA + HFD groups) showed significant higher levels of total serum cholesterol, HDL and triglycerides than healthy rabbits $(P=0.01)$. Likewise, the AIA + HFD group had higher lipid levels compared with the AIA group (Figure 1B). A decrease of rabbit weight $(\mathrm{kg})$ in the HFD, AIA and AIA + HFD groups $(3.4 \pm 0.19 ; 3.23 \pm 0.22$ and $3.28 \pm 0.12)$ was observed when compared to healthy rabbits $(4.28 \pm 0.06$; $P<0.002)$, at the time of sacrifice.

\section{CRP levels}

Levels of CRP were significantly increased in sera of AIA and AIA + HFD groups (73.07 \pm 32.58 and $124.2 \pm 38.69)$ in comparison with the healthy group $(7.83 \pm 1.28, P=$ 0.011 and $P<0.001$, respectively). In addition, CRP levels of the AIA + HFD group were higher than the HFD group $(124.2 \pm 38.69$ versus $35.71 \pm 13.98, P=0.042)$. No significant difference was found between the AIA and AIA + HFD groups; however, an increased tendency is observed in the AIA + HFD group (Figure 1C).

\section{Increase of synovial inflammation}

Histological changes of the synovial membrane were evaluated by the Krenn synovitis score. Synovial lesions were observed in HFD group compared with healthy rabbits $(P=0.005)$. AIA and AIA + HFD rabbits showed much more severe synovial changes than control groups, including lining hyperplasia, mononuclear cell infiltration at the sublining layer and lymphoid aggregates, occasionally organized into nodule-like structures (Figure 2). These synovial features mirror those present in rheumatoid synovium [29].

\section{Massive $\mathbf{M} \phi$ infiltration and presence of foam cells}

Although both AIA and AIA + HFD rabbits presented the highest synovitis score, AIA + HFD synovium was characterized by a massive infiltration of RAM11-positive cells whose presence was greater than AIA and control synovial membranes $(P=0.001$; Figure $3 \mathrm{E})$. This remarkable feature along with the notable and consistent presence of foam cells in AIA + HFD synovium (Figures $2 \mathrm{~F}$ and $3 \mathrm{~F}$ ), detected by Oil Red-O staining (data not shown), demonstrates a clear qualitative difference between synovial membranes of similar inflammation score grade from the AIA + HFD and AIA groups. The presence of foam cells was detected in the sublining and lining layers; the latter may be considered as a pathognomonic feature of the AIA + HFD group.

\section{Increase of TNF- $\alpha$ and MCP-1 gene expression}

The HFD, AIA and AIA + HFD groups showed a threefold increase of TNF- $\alpha$ gene expression levels (3.57 \pm $0.73 ; 3.17 \pm 1.25$ and $2.44 \pm 0.26)$ when compared with healthy rabbits $(1.16 \pm 0.24 ; P=0.005 ; P=0.05$ and $P=$ 0.005). MCP-1 gene expression levels of HFD and AIA groups were also increased when compared to the healthy group $(2.59 \pm 0.54 ; 3.50 \pm 1.42$ vs. $1.04 \pm 0.17$; $P=0.006 ; P=0.016)$. AIA + HFD MCP-1 gene expression levels were lower than AIA $(1.54 \pm 0.22$ vs. $3.50 \pm$ 1.42; $P=0.048$ ) (Additional file 1).

\section{Presence of multinucleated cells resorbing adipose tissue} Macrophages arranged in crown-like structures around adipocytes were observed in HFD, AIA and AIA + HFD synovium (Figure 4A, B). Interestingly, we found more frequently multinucleated cells engulfing adipocyte structures in the AIA + HFD group (Figure 4B), and even more progression of multinucleated cells, going from two up to multiple nuclei (Figure 4C). The number of multinucleated cells was significantly increased in AIA + HFD synovium $(P<0.001$; Figure 4D). Circulating synovial multinucleated cells were also observed in the proximity of the region, some of them were cathepsin K-positive (Figure 4E).

\section{Increase of angiogenesis}

Immunohistochemical analysis of synovial membranes for CD31 confirmed an increased vascularization by a higher number of vessels stained in AIA + HFD synovium compared with AIA synovium. Both groups showed by far more CD31-positive vessels than the HFD and healthy groups (Figure 5A, E). In addition, no 


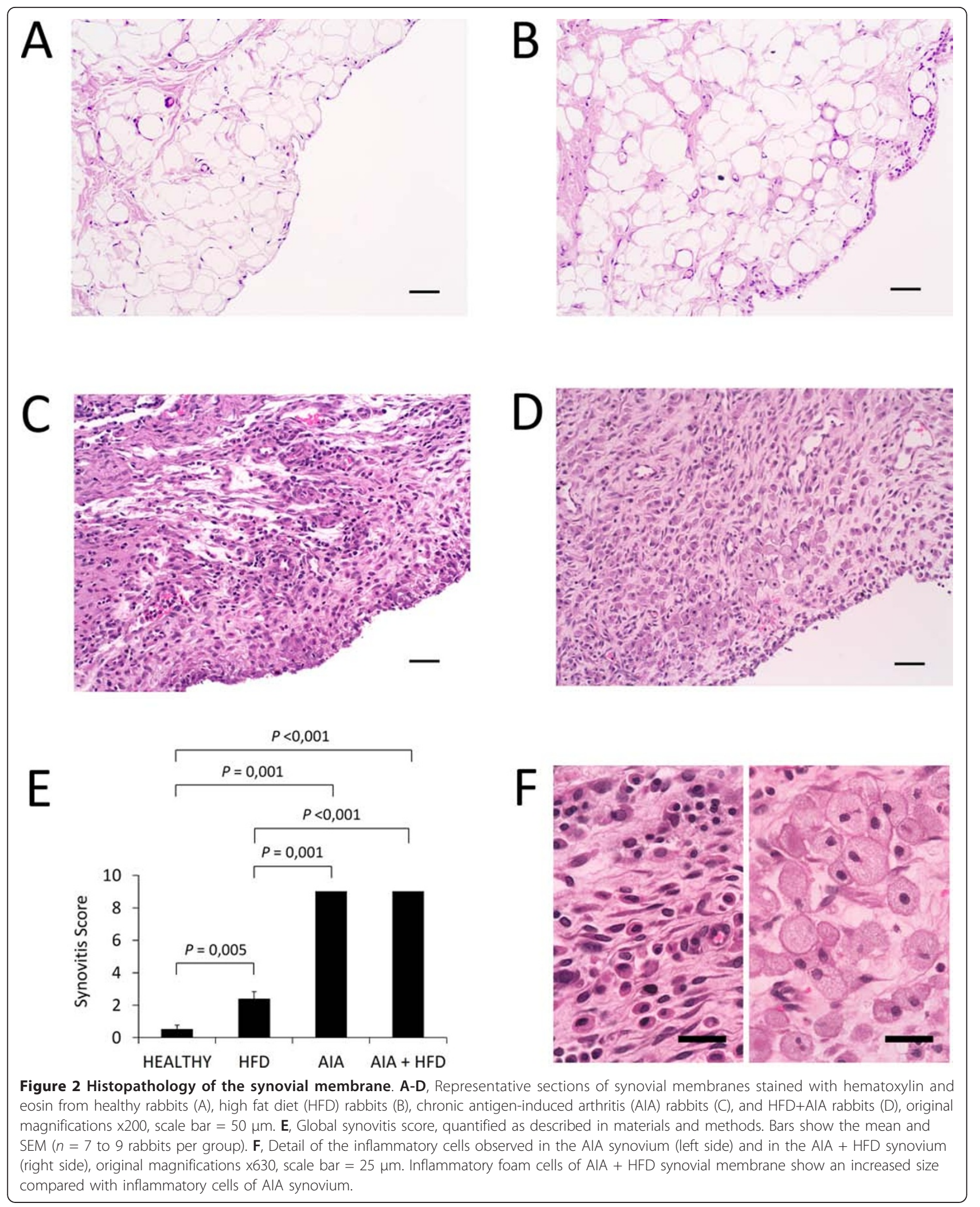




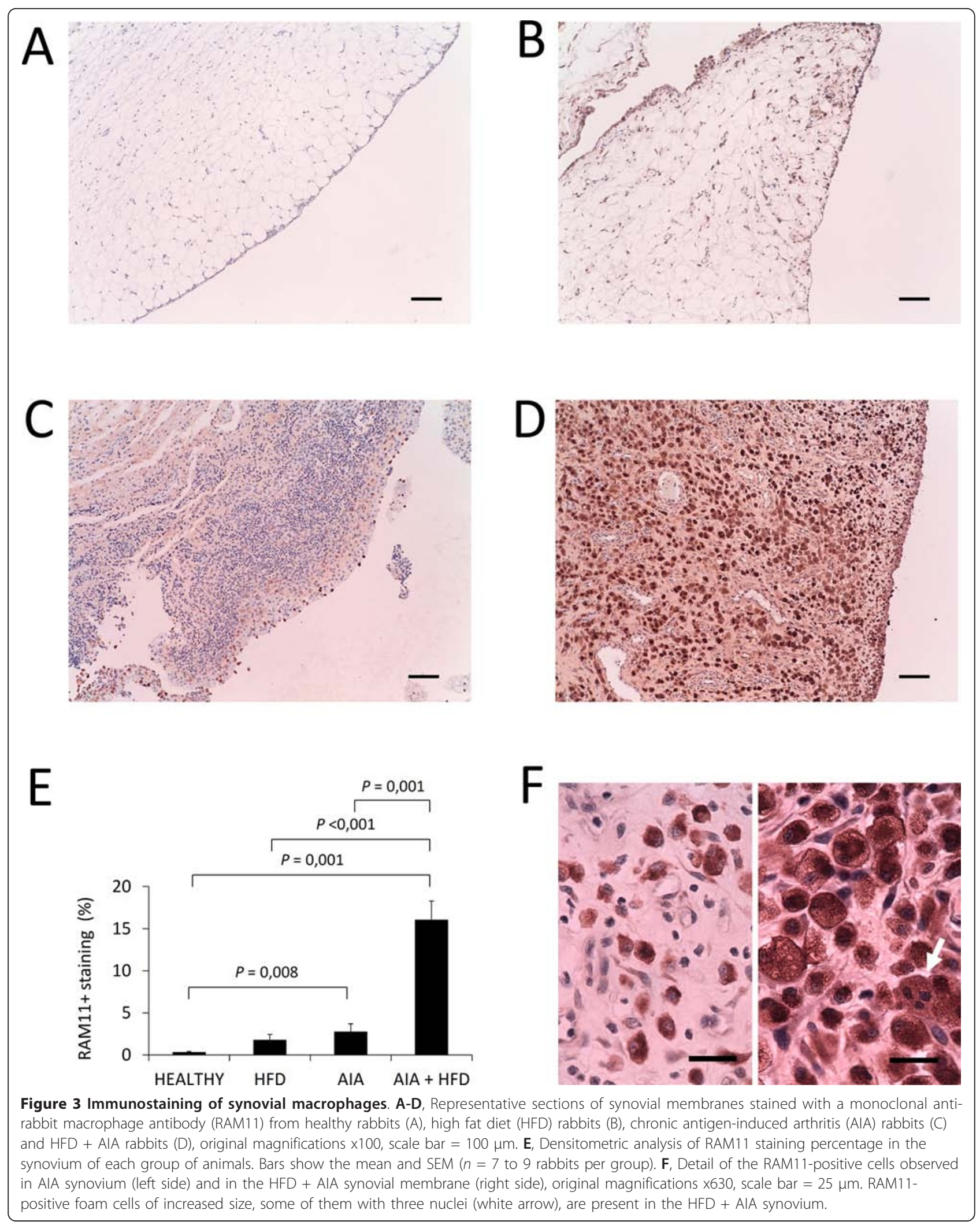




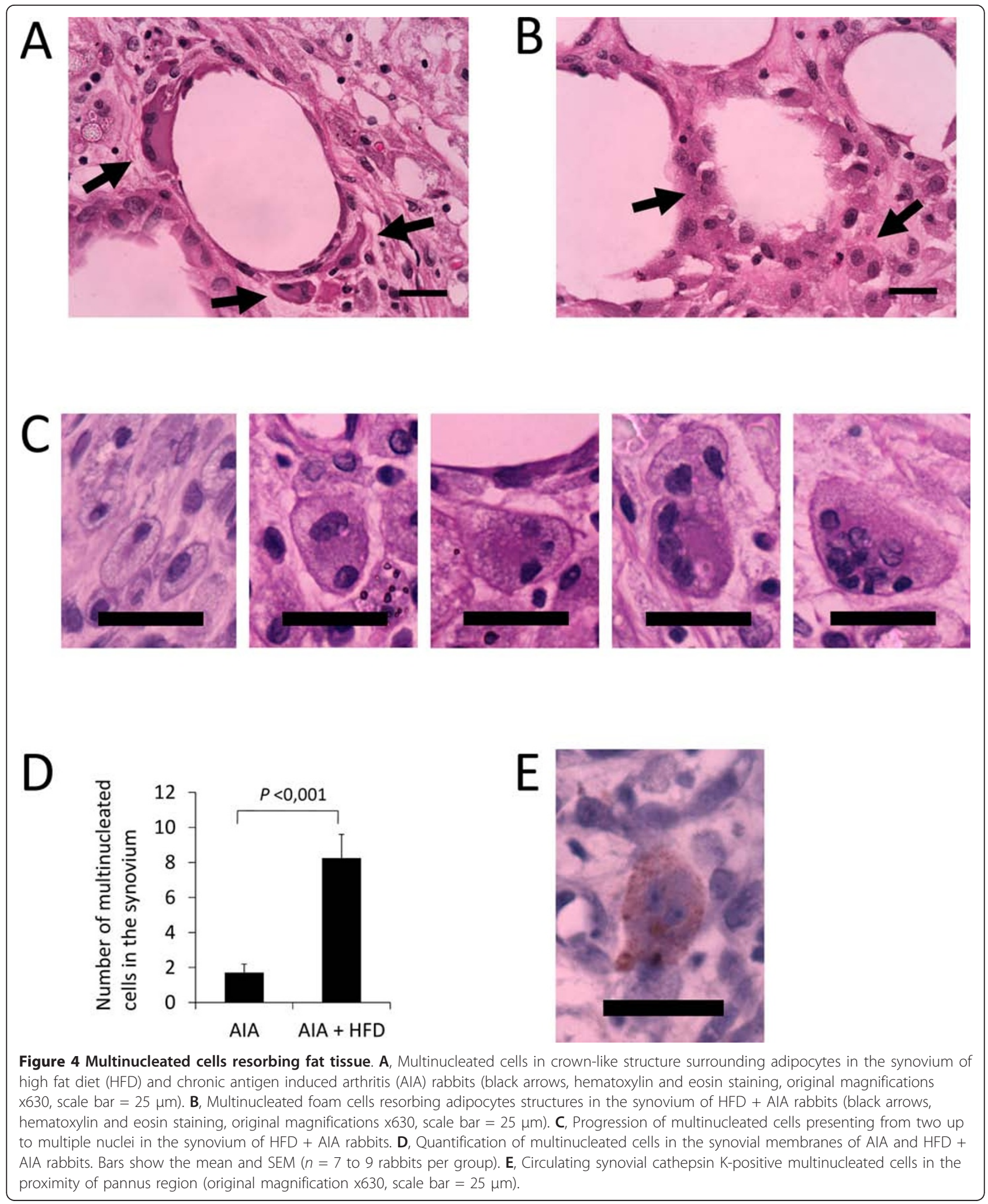

difference was observed for CD31 staining between healthy and HFD groups; however, a tendency of more CD31 staining can be observed in HFD synovium.

\section{Increase of active bone resorption}

We estimated active bone resorption by quantifying cathepsin K-positive osteoclasts and by determining the 


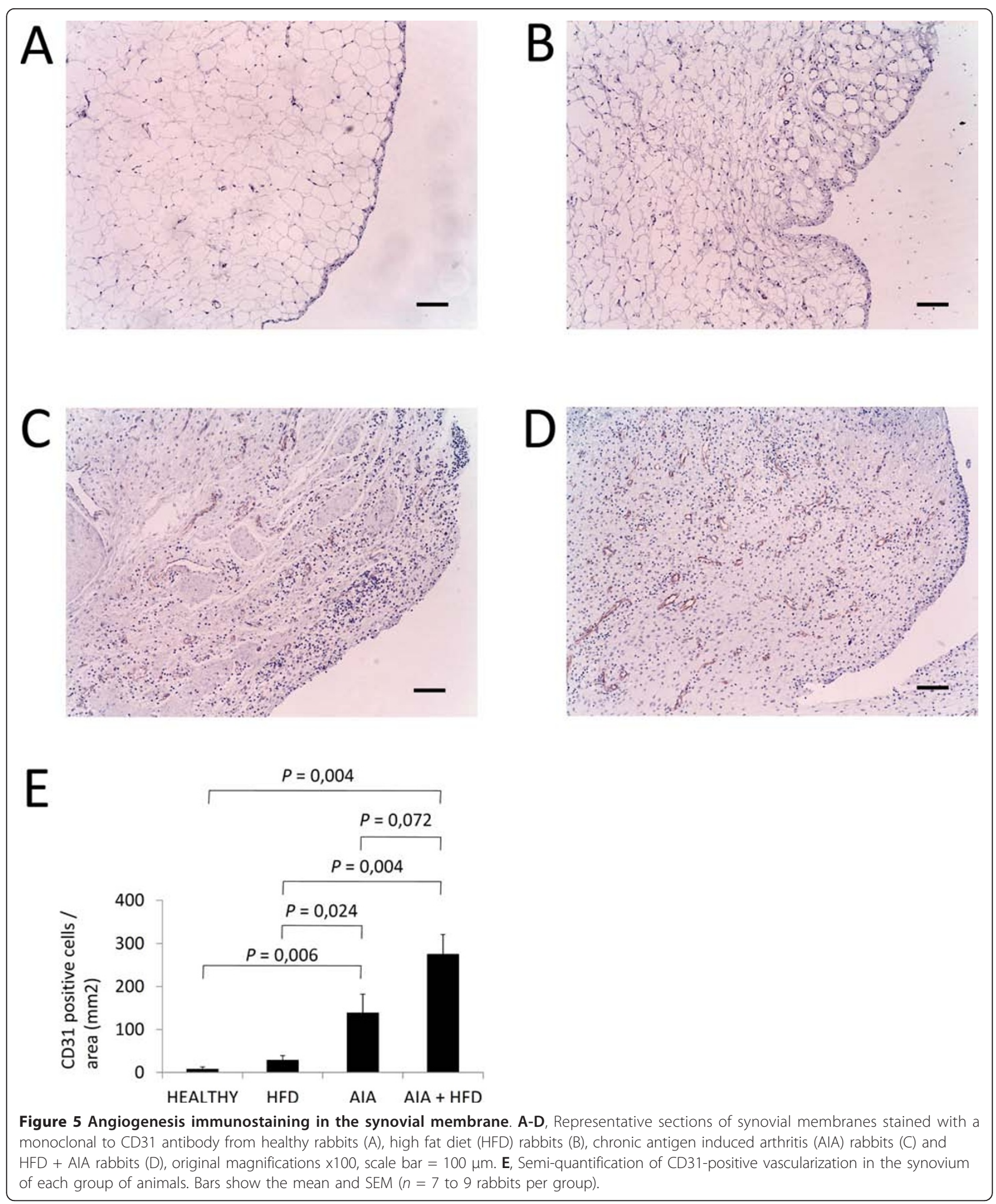

contact surface between pannus and bone/calcified cartilage and the area of invasive pannus into bone. Our results showed an increased presence of cathepsin K- positive osteoclasts in the synovium of AIA + HFD rabbits compared with that of AIA rabbits $(P=0.015$; Figure 6A, B). We also observed a higher surface of 


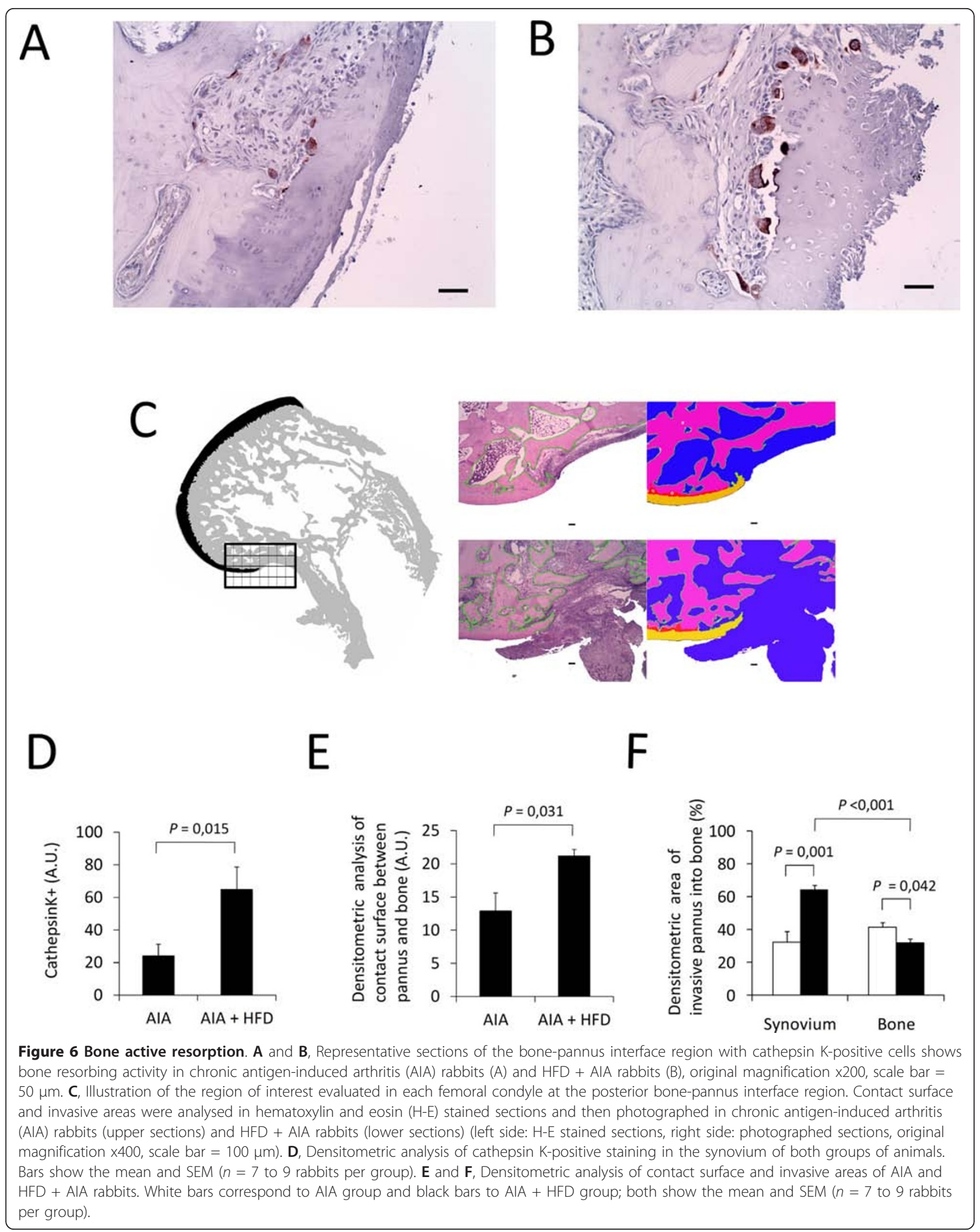


bone resorbing pannus in AIA + HFD than in AIA rabbits (Figure 6C, F). We selected the posterior pannusbone area as the region of interest to be measured because osteoclast differentiation from macrophages and activation within the aggressive pannus and subsequent subchondral bone invasion occur in this interface.

\section{Increase of RANKL protein levels}

Both RANKL and OPG are expressed in cartilage, bone and synovium, as previously described [30]. RANKL expression in the HFD group was increased in all tissues when compared to the healthy group $(2.49 \pm 0.29$ vs. $0.99 \pm 0.18, P=0.001$ in cartilage; $4.10 \pm 0.46$ vs. $2.26 \pm$ $0.34, P=0.021$ in bone; and $7.06 \pm 1.01$ vs. $3.04 \pm 0.34$, $P=0.015$ in synovium). RANKL expression levels in both AIA and AIA + HFD groups were highly increased in all tissues when compared with the controls, healthy and HFD groups. Specifically in the synovium, AIA and AIA + HFD groups showed similar levels of RANKL protein expression $(24.79 \pm 2.23$ and $24.63 \pm 2.05)$ and were significantly higher compared with their controls, healthy and HFD groups $(3.04 \pm 0.34, P<0.001$ and $7.06 \pm 1.01$, $P<0.001$, respectively) (Additional file 2 ).

\section{Discussion}

This is the first in vivo study that demonstrates the harmful effect of hypercholesterolemia on the severity of chronic arthritis. Indeed, we characterized a novel model of chronic arthritis aggravated by hypercholesterolemia, which is driven by foam $\mathrm{M} \phi$ with an aggressive phenotype. These $\mathrm{M} \phi$ markedly damaged synovial membrane, calcified cartilage, juxta-articular bone and fatty tissue, and increased angiogenesis was also evidenced in the synovial membrane of our experimental animals. Likewise, reduced total cholesterol and HDL levels, as well as increased lowdensity lipoprotein (LDL)/vLDL, IL-6, sRANKL and CCL5 circulating levels mirroring RA patients was observed in the $K / B x A g 7$ mice, a novel animal model of erosive arthritis followed by prominent aortic atherosclerosis. Remarkably, etanercept administration reduced arthritis and atherosclerosis development in these mice [31].

We found a modest, although evident, increase of synovial cell infiltration in non-arthritic rabbits fed with hypercholesterolemic diet. Accordingly, an atherogenic lipid profile characterized by high total cholesterol, triglyceride and apo B levels, and low HDLc levels was more prevalent in blood donors who later developed RA [32]. Similarly, a higher risk of moderate to severe RA was found in patients with metabolic syndrome [33]. Recent obesity studies have supported these findings since the lipid profile may also be influenced by weight/body mass index. Obesity has been associated with a modest risk for developing RA, but the rapid increase in obesity prevalence may be responsible for much of the increase in RA incidence [34]. Moreover, obesity was associated with worse RA disease outcomes, high prevalence of comorbidities and the poor therapy effect of glucocorticoids, disease-modifying antirheumatic drugs (DMARDs) and biologic agents [35-37]. All these findings suggest that hyperlipidemia may influence the initiation and progression of chronic inflammatory synovitis as well as indicate the potential relevance of its pharmacology modification.

Since there are no clearly designed studies showing that hypercholesterolemia increases chronic synovitis, investigating the effects of hypolipidemic statins on the course of RA may provide additional clues to this relationship. Besides their well-established beneficial effects on lipid metabolism in patients with hypercholesterolemia, cardiovascular diseases and inflammatory diseases [38-40], statins have also been shown to exert anti-inflammatory, antioxidant, immunomodulatory and antithrombotic effects [41]. A six-month clinical trial reported a modest but significant clinical improvement in RA subjects treated with atorvastatin [39]. Interestingly, a recent large population-based cohort study has shown an association between good adherence to statin treatment and reduced risk of developing RA [42]. Likewise, statins reduced the risk for the development of RA between $30 \%$ and $40 \%$, although lipid lowering drugs other than statins were not associated with a decreased risk [43]. Hence, statins may be protective against development and progression of RA in those with hyperlipidemia.

Massive infiltrates of RAM11-positive macrophages, a major number of multinucleated cells and the presence of foam-like cells were characteristically seen in the AIA + HFD synovium. Activation of blood mononuclear cells as shown by enhanced nuclear factor-kappa B (NF- $\kappa$ B) transcription binding and massive $\mathrm{M} \phi$ infiltration in atherosclerotic plaques was previously described in the combined model [16]. Taken together, these findings suggest that the activation of peripheral monocytes could lead to systemic inflammation producing a huge $\mathrm{M} \phi$ tissue infiltration, accounting for the synovitis aggressiveness observed in this experimental model. In accordance, several quantitative immunohistological studies have highlighted the relevance of $\mathrm{M} \phi$ in driving the inflammatory response. The number of $\mathrm{M} \phi$ was increased in clinically affected joints compared with non-affected joints [44], and correlated well with clinical signs of disease activity, including joint pain and swelling [18]. Of relevance, the change in the number of sublining $\mathrm{M} \phi$ has been proposed as a sensitive biomarker to predict possible efficacy of new anti-rheumatic treatment [45].

The high plasticity of macrophages, as shown by the marked transformation of macrophages into foam cells, lipid-engulfed multinucleated cells and further cathepsin K-stained osteoclasts, was one of the major findings in hyperlipidemic rabbits with chronic arthritis. As described 
in the adipose tissue of obese mice and humans [17,46], macrophages were frequently observed around dead adipocytes forming characteristic 'crown-like' structures. This shows an exacerbated phagocytosis of dead adipocytes by activated macrophages in the sublining of AIA + HFD rabbits. The increased transformation of activated macrophages into foam cells in the lining and sublining layers of hyperlipemic animals with AIA occurs as a result of the enhanced uptake by their scavenger receptors of oxidized low density lipoproteins (oxLDL). A disruption of the transcriptional equilibrium mediated by lipid sensors between lipid metabolism and immune functions in macrophages promotes the enhancement of pro-inflammatory signals during their activation, and finally leads to formation of foam cells [17]. A persistent imbalance between pro- and anti-inflammatory mediators released by macrophages and/or deficient clearance of apoptotic foam cells can perpetuate chronic inflammation [47]. Indeed, macrophages from experimental animals generated significant levels of TNF- $\alpha$ gene expression and, potentially, other inflammatory mediators, such as IL-6 signaling, that may have induced C-reactive protein expression [16].

The growth of the synovial membrane was accompanied by neovascularization, as demonstrated by the increased CD31+ staining of blood vessels in AIA and, particularly, in AIA + HFD synovial membranes. Activated macrophages promote angiogenesis by the production of angiogenic factors, including pro-inflammatory cytokines, growth factors and chemokines that promote endothelial cell adhesion, receptor expression and neovascularization [48]. Many of these molecules were not induced in adipose tissue macrophages from $\mathrm{C}$-C chemokine receptor 2 (CCR2) knockout mice fed with a high-fat diet, supporting the importance of CCR2 in regulating recruitment of inflammatory activated macrophages during obesity [49]. Successful treatment of RA with anti-TNF antibodies reduced levels of pro-angiogenic factors, including vascular endothelial growth factor (VEGF), and led to normalization of the vasculature. In addition, leptin has been suggested to induce angiogenesis in endochondral ossification [50]. These data emphasize the close links among angiogenesis, inflammation and obesity in this disease. In our animal model, whether hypercholesterolemia boosts angiogenesis in the synovium of arthritic rabbits remains to be established and ongoing research is being carried out in this regard.

The phenotype transformation of macrophages to cathepsin K-stained osteoclasts observed in AIA and, particularly, in AIA rabbits fed with a hyperlipemic diet remarkably resembles the development of osteoclasts from monocytes/macrophage precursor cells, one of the unique characteristic of RA [7]. Cathepsin $\mathrm{K}$ is the major protease of osteoclasts responsible for bone resorption and its specific degradation product, $\mathrm{C}$-terminal telopeptide of collagen type I (CTX-I), has been extensively used as a surrogate measure of bone resorption [7,10,51-53]. Thus, the high active bone resorption found in AIA + HFD synovial membranes shows the influence of hyperlipidemia on bone metabolism. In fact, human macrophage foam cells produce CTX-I fragments in atherosclerotic plaques $[19,20,22,23,53,54]$. Multiple parameters of bone quality were altered by a hypercholesterolemic diet in mice, including bone mineral density (BMD) bone volume fraction, number of trabeculae and trabecular spacing, along with changes in the mechanical properties of the bone and an increase in bone resident osteoclasts [24]. Although synovitis score and the expression levels of RANKL, OPG and TNF- $\alpha$ were similar in AIA and AIA + HFD groups, bone resorption activity was remarkably increased in AIA + HFD rabbits suggesting a greater RANKL activity or efficiency in the presence of high LDL levels. It has recently been described that LDL deficiency causes impaired osteoclastogenesis with a subsequent decrease in bone resorption parameters and increased bone mass in mice due to a defect in osteoclastic cell-cell fusion [55]. In addition, Wnt-mediated signals might be altered in hyperlipidemia and, subsequently, affect bone resorption [56]. Thus, hypercholesterolemia may directly disrupt bone homeostasis.

\section{Conclusions}

These findings provide a valuable piece of information about the hypercholesterolemia pathogenicity at the synovial membrane and cartilage-pannus junction. The hyperlipidemia-boosted systemic inflammation markedly damages articular tissues by macrophage massive infiltration and transformation to foam cells and active osteoclasts. In this context, $\mathrm{M} \phi$ accumulation at the site of inflammation may be a direct response to the abnormal fat metabolism caused by the increasing adiposity. Therefore, this experimental model shows that hypercholesterolemia markedly increments joint tissue damage in chronic arthritis, demonstrating a key role of synovial macrophages in this process, as well as their potential as a suitable target for successful RA therapy.

\section{Additional material}

Additional file 1: High fat diet (HFD) and antigen induced arthritis
(AIA) interventions increase TNF- $\alpha$ (A) and MCP-1 (B) gene
expression in the synovium. Gene expression was examined by real
time PCR and results are expressed as fold induction. Bars show the
mean and SEM ( $n=7$ to 9 rabbits per group).
Additional file 2: RANKL and OPG protein expression. A, C and E,
Densitometric analysis of receptor activator nuclear kappa B ligand
(RANKL) and osteoprotegerin (OPG) protein expression in cartilage (A),
subchondral bone (C) and synovium (E). Bars show the mean and SEM
( $n=7$ to 9 rabbits per group). B, D and $\mathbf{F}$, Representative Western blot
images for RANKL and OPG detection in cartilage (B), subchondral bone
(C) and synovium (F). EZ Blue-stained gels used as protein loading
(AIA) interventions increase TNF- $\alpha$ (A) and MCP-1 (B) gene expression in the synovium. Gene expression was examined by real time PCR and results are expressed as fold induction. Bars show the mean and SEM ( $n=7$ to 9 rabbits per group). Densitometric analysis of receptor activator nuclear kappa $B$ ligand (RANKL) and osteoprotegerin (OPG) protein expression in cartilage (A), ( $n=7$ to 9 rabbits per group). B, D and $\mathbf{F}$, Representative Western blot (C) and synovium (F). EZ Blue-stained gels used as protein loading 
controls are also shown for healthy rabbits, high fat diet (HFD) rabbits, chronic antigen induced arthritis (AIA) rabbits and HFD + AIA rabbits.

\section{Abbreviations}

AIA: antigen induced arthritis; apoB: apolipoprotein B; BMD: bone mineral density; CCL5: C-C chemokine ligand 5; CCR2: C-C chemokine receptor 2; CRP: C reactive protein; CTX1: C terminal telopeptide of collagen type I; DMARDs: disease-modifying anti-rheumatic drugs; EDTA: ethylenediaminetetraacetic acid; GAPDH: glyceraldehydes 3-phosphate dehydrogenase; HDL: high density lipoprotein; H-E: hematoxylin and eosin; HEPES: hydroxyethylpiperazine-1-ethanesulfonic acid; HFD: high fat diet; IgG: immunoglobulin G; IL6: interleukin 6; LDL: low density lipoprotein; M1: proinflammatory macrophage; M2: anti-inflammatory macrophage; MCP-1: monocyte chemoattractant protein 1; MФ: macrophage; NFkB: nuclear factor kappa B; NP: nonyl phenoxypolyethoxylethanol; OPG: osteoprotegerin; OVA: ovalbumin; OxLDL: oxidized low density lipoprein; PBS: phosphate buffered saline; PMSF: phenylmethanesulfonylfluoride; PVDF: polyvinylidene difluoride; RA: rheumatoid arthritis; RAM11: anti-rabbit macrophage monoclonal antibody; RANKL: receptor activator nuclear kappa B ligand; SDS: sodium dodecyl sulfate; SEM: standard error mean; SRANKL: soluble receptor activator nuclear kappa B ligand; TNF-a: tumor necrosis factor a; VEGF: vascular endothelial growth factor; $V L D L$ : very low density lipoprotein

\section{Competing interests}

The authors declare that they have no competing interests.

\section{Authors' contributions}

$G H-B, J A R-B, I P-P$ and $R L$ were involved in the study conception and design. IP-P, MJM-C and RG were involved in the acquisition of data. IP-P, JAR-B, $M J M-C, R G, R L$ and $G H-B$ were involved in the analysis and interpretation of data. GH-B had full access to all study data and takes responsibility for the integrity and accuracy of data analysis. All authors were involved in drafting the article or revising it critically for important intellectual content. All authors approved the final version to be published.

\section{Acknowledgements}

This work was supported by research grants from Instituto de Salud Carlos III (PS09/01625, GH-B and PS09/00034, RL). IP-P is the recipient of a fellowship from Fundacion Conchita Rábago. MJM-C and RG are both recipients of a Sara Borrell contract from Instituto de Salud Carlos III. RL was funded by Instituto de Salud Carlos III through a research staff stabilization program.

Received: 25 February 2013 Revised: 23 May 2013

Accepted: 13 August 2013 Published: 13 August 2013

\section{References}

1. Gremese E, Ferraccioli G: The metabolic syndrome: the crossroads between rheumatoid arthritis and cardiovascular risk. Autoimmun Rev 2011, 10:582-589.

2. Gabriel S: The epidemiology of rheumatoid arthritis. Rheum Dis Clin North Am 2001, 27:269-281.

3. Romero Fl, Martínez-Calatrava MJ, Sánchez-Pernaute O, Gualillo O, Largo R, Herrero-Beaumont G: Pharmacological modulation by celecoxib of cachexia associated with experimental arthritis and atherosclerosis in rabbits. Br J Pharmacol 2010, 161:1012-1022.

4. Scotece M, Conde J, Gómez R, López V, Pino J, González A, Lago F, GómezReino JJ, Gualillo O: Role of adipokines in atherosclerosis: interferences with cardiovascular complications in rheumatic diseases. Mediators Inflamm 2012, 2012:125458.

5. Lago R, Gómez R, Lago F, Gómez-Reino J, Gualillo O: Leptin beyond body weight regulation-current concepts concerning its role in immune function and inflammation. Cell Immunol 2008, 252:139-145.

6. Gómez R, Conde J, Scotece M, Gómez-Reino JJ, Lago F, Gualillo O: What's new in our understanding of the role of adipokines in rheumatic diseases? Nat Rev Rheumatol 2011, 7:528-536.

7. Li J, Hsu H-C, Mountz JD: Managing macrophages in rheumatoid arthritis by reform or removal. Curr Rheumatol Rep 2012, 14:445-454.
8. Xu H, Barnes GT, Yang Q, Tan G, Yang D, Chou CJ, Sole J, Nichols A, Ross JS, Tartaglia LA, Chen H: Chronic inflammation in fat plays a crucial role in the development of obesity-related insulin resistance. J Clin Invest 2003, 112:1821-1830

9. Libby P, Ridker PM, Hansson GK: Progress and challenges in translating the biology of atherosclerosis. Nature 2011, 473:317-325.

10. Szekanecz Z, Koch AE: Vasculogenesis in rheumatoid arthritis. Arthritis Res Ther 2010, 12:110.

11. Jodon de Villeroché V, Avouac J, Ponceau A, Ruiz B, Kahan A, Boileau C, Uzan G, Allanore Y: Enhanced late-outgrowth circulating endothelial progenitor cell levels in rheumatoid arthritis and correlation with disease activity. Arthritis Res Ther 2010, 12:R27.

12. Paleolog E: It's all in the blood: circulating endothelial progenitor cells link synovial vascularity with cardiovascular mortality in rheumatoid arthritis? Arthritis Res Ther 2005, 7:270-272.

13. Shantsila E, Watson T, Tse HF Lip GY: New insights on endothelial progenitor cell subpopulations and their angiogenic properties. J Am Coll Cardiol 2008, 12:669-671.

14. Weber $\mathrm{C}$, Noels $\mathrm{H}$ : Atherosclerosis: current pathogenesis and therapeutic options. Nat Med 2011, 17:1410-1422.

15. Crowther M: Pathogenesis of atherosclerosis. Hematology Am Soc Hematol Educ Program 2005, 436-441.

16. Largo R, Sánchez-Pernaute O, Marcos ME, Moreno-Rubio J, Aparicio C, Granado R, Ortega L, Egido J, Herrero-Beaumont G: Chronic arthritis aggravates vascular lesions in rabbits with atherosclerosis: a novel model of atherosclerosis associated with chronic inflammation. Arthritis Rheum 2008, 58:2723-2734.

17. EM and Monach P: The rheumatoid joint: synovitis and tissue destruction. Rheumatology. 5 edition. Philadelphia, PA: Mosby-Elsevier; 2011, 911-934.

18. Tak PP, Smeets TOMJM, Daha MR, Kluin PM, Meijers KAE, Brand R, Meinders AED, Breedveld FC: Analysis of the synovial cell infiltrate in relation to local disease activity. Arthritis Rheum 1997, 40:217-225.

19. De Rycke L, Baeten D, Foell D, Kruithof E, Veys EM, Roth J, De Keyser F: Differential expression and response to anti-TNFalpha treatment of infiltrating versus resident tissue macrophage subsets in autoimmune arthritis. J Pathol 2005, 206:17-27.

20. Tak PP, Bresnihan B: The pathogenesis and prevention of joint damage in advances from synovial biopsy and tissue analysis. Arthritis Rheum 2000, 43:2619-2633.

21. Bonet ML, Granados N, Palou A: Molecular players at the intersection of obesity and osteoarthritis. Curr Drug Targets 2011, 12:2103-2128.

22. Prieur $X$, Mok CY, Velagapudi VR, Núñez V, Fuentes $L$, Montaner $D$, Ishikawa K, Camacho A, Barbarroja N, O'Rahilly S, Sethi JK, Dopazo J, Orešič M, Ricote M, Vidal-Puig A: Differential lipid partitioning between adipocytes and tissue macrophages modulates macrophage lipotoxicity and M2/M1 polarization in obese mice. Diabetes 2011, 60:797-809.

23. Tintut $Y$, Morony S, Demer LL: Hyperlipidemia promotes osteoclastic potential of bone marrow cells ex vivo. Arterioscler Thromb Vasc Biol 2004, 24:e6-10.

24. Pelton K, Krieder J, Joiner D, Freeman MR, Goldstein SA, Solomon KR: Hypercholesterolemia promotes an osteoporotic phenotype. Am J Pathol 2012, 181:1-9.

25. Alvarez-Soria M, Largo R, Santillana J, Sánchez-Pernaute O, Calvo E, Hernández M, Egido J, Herrero-Beaumont G: Long term NSAID treatment inhibits COX-2 synthesis in the knee synovial membrane of patients with osteoarthritis: differential proinflammatory cytokine profile between celecoxib and aceclofenac. Ann Rheum Dis 2006, 65:998-1005.

26. Krenn V, Morawietz L, Häupl T, Neidel J, Petersen I, König A: Grading of chronic synovitis - a histopathological grading system for molecular and diagnostic pathology. Pathol Res Pract 2002, 198:317-325.

27. Herrero-Beaumont $G$, Marcos ME, Sánchez-Pernaute $O$, Granados $R$, Ortega L, Montell E, Vergés J, Egido J, Largo R: Effect of chondroitin sulphate in a rabbit model of atherosclerosis aggravated by chronic arthritis. Br J Pharmacol 2008, 154:843-851.

28. Bellido M, Lugo L, Roman-Blas JA, Castañeda S, Caeiro JR, Dapia S, Calvo E, Largo R, Herrero-Beaumont G: Subchondral bone microstructural damage by increased remodelling aggravates experimental osteoarthritis preceded by osteoporosis. Arthritis Res Ther 2010, 12:R152.

29. Hitchon C, El-Gabalawy HS: The synovium in rheumatoid arthritis. Open Rheumatol I 2011, 5:107-114. 
30. Martínez-Calatrava MJ, Prieto-Potín I, Roman-Blas JA, Tardio L, Largo R, Herrero-Beaumont G: RANKL synthesized by articular chondrocytes contributes to juxta-articular bone loss in chronic arthritis. Arthritis Res Ther 2012, 14:R149.

31. Rose S, Eren M, Murphy S, Zhang H, Thaxton CS, Chowaniec J, Waters EA, Meade TJ, Vaughan DE, Perlman H: A novel mouse model that develops spontaneous arthritis and is predisposed towards atherosclerosis. Ann Rheum Dis 2012, 72:89-95.

32. van Halm VP, Nielen MM, Nurmohamed MT, van Schaardenburg $D$, Reesink HW, Voskuyl AE, Twisk JW, van de Stadt RJ, de Koning MH, Habibuw MR, van der Horst-Bruinsma IE, Dijkmans BA: Lipids and inflammation: serial measurements of the lipid profile of blood donors who later developed rheumatoid arthritis. Ann Rheum Dis 2007, 66:184-188.

33. Karvounaris SA, Sidiropoulos PI, Papadakis JA, Spanakis EK, Bertsias GK, Kritikos HD, Ganotakis ES, Boumpas DT: Metabolic syndrome is common among middle-to-older aged Mediterranean patients with rheumatoid arthritis and correlates with disease activity: a retrospective, crosssectional, controlled, study. Ann Rheum Dis 2007, 66:28-33.

34. Crowson CS, Matteson EL, Davis JM, Gabriel SE: Contribution of obesity to the rise in incidence of rheumatoid arthritis. Arthritis Res Ther 2013, 65:71-77.

35. Ajeganova S, Andersson ML, Hafström I: Association of obesity with worse disease severity in rheumatoid arthritis as well as with comorbidities: a long-term followup from disease onset. Arthritis Care Res 2013, 65:78-87.

36. Klaasen R, Wijbrandts C, Gerlag DM, Tak PP: Body mass index and clinical response to infliximab in rheumatoid arthritis. Arthritis Rheum 2011, 63:359-364.

37. Gremese E, Carletto A, Padovan M, Atzeni F, Raffeiner B, Giardina AR, Favalli EG, Erre GL, Gorla R, Galeazzi M, Foti R, Cantini F, Salvarani C, Olivieri I, Lapadula G, Ferraccioli G, Gruppo Italiano di Studio sulle Early Arthritis (GISEA): Obesity and reduction of the response rate to antitumor necrosis factor $a$ in rheumatoid arthritis: an approach to a personalized medicine. Arthritis Care Res (Hoboken) 2013, 65:94-100

38. Baigent C, Keech A, Kearney PM, Blackwell L, Buck G, Pollicino C, Kirby A, Sourjina T, Peto R, Collins R, Simes R, Cholesterol Treatment Trialists' (CTT) Collaborators: Efficacy and safety of cholesterol-lowering treatment: prospective meta-analysis of data from 90,056 participants in 14 randomised trials of statins. Lancet 2005, 366:1267-1278.

39. McCarey DW, McInnes IB, Madhok R, Hampson R, Scherbakova O, Ford I, Capell HA, Sattar N: Trial of Atorvastatin in Rheumatoid Arthritis (TARA): double-blind, randomised placebo-controlled trial. Lancet 2004, 363:2015-2021.

40. Paraskevas Kl: Statin treatment for rheumatoid arthritis: a promising novel indication. Clin Rheumatol 2008, 27:281-287.

41. Lopez-Pedrera C, Ruiz-Limon P, Valverde-Estepa A, Barbarroja N, RodriguezAriza A: To cardiovascular disease and beyond: new therapeutic perspectives of statins in autoimmune diseases and cancer. Curr Drug Targets 2012, 13:829-841.

42. Chodick G, Amital H, Shalem Y, Kokia E, Heymann AD, Porath A, Shalev V: Persistence with statins and onset of rheumatoid arthritis: a populationbased cohort study. PLoS Med 2010, 7:e1000336.

43. Jick SS, Choi H, Li L, McInnes IB, Sattar N: Hyperlipidemia, statin use and the risk of developing rheumatoid arthritis. Ann Rheum Dis 2009, 68:546-551.

44. Kraan MC, Versendaal H, Jonker M, Bresnihan B, Post WJ, t Hart BA, Breedveld FC, Tak PP: Asymptomatic synovitis precedes clinically manifest arthritis. Arthritis Rheum 1998, 41:1481-1488.

45. Haringman JJ, Gerlag DM, Zwinderman AH, Smeets TJ, Kraan MC, Baeten D, Mclnnes IB, Bresnihan B, Tak PP: Synovial tissue macrophages: a sensitive biomarker for response to treatment in patients with rheumatoid arthritis. Ann Rheum Dis 2005, 64:834-838.

46. Murano I, Barbatelli G, Parisani V, Latini C, Muzzonigro G, Castellucci M: Dead adipocytes, detected as crown-like structures, are prevalent in visceral fat depots of genetically obese mice. J Lipid Res 2008, 49:1562-1568.

47. Dushkin MI: Macrophage/foam cell is an attribute of inflammation: mechanisms of formation and functional role. Biochemistry (Mosc) 2012, 77:327-38.

48. Szekanecz Z, Koch AE: Vascular involvement in rheumatic diseases: "vascular rheumatology". Arthritis Res Ther 2008, 10:224.
49. Lumeng CN, Deyoung SM, Bodzin JL, Saltiel AR: Increased inflammatory properties of adipose tissue macrophages recruited during diet-induced obesity. Diabetes 2007, 56:16-23.

50. Kume K, Satomura K, Nishisho S, Kitaoka E, Yamanouchi K, Tobiume S, Nagayama M: Potential role of leptin in endochondral ossification. $J$ Histochem Cytochem 2002, 50:159-169.

51. Chase J: Role of nuclear factor-kappaB activation in metalloproteinase-1, -3 , and -9 secretion by human macrophages in vitro and rabbit foam cells produced in vivo. Arterioscler Thromb Vasc Biol 2002, 22:765-771.

52. Mosser DM, Edwards JP: Exploring the full spectrum of macrophage activation. Nat Rev Immunol 2008, 8:958-969.

53. Barascuk N, Skjøt-Arkil H, Register TC, Larsen L, Byrjalsen I, Christiansen C, Karsdal MA: Human macrophage foam cells degrade atherosclerotic plaques through cathepsin $\mathrm{K}$ mediated processes. BMC Cardiovasc Disord 2010, 10:19.

54. Weisberg SP, McCann D, Desai M, Rosenbaum M, Leibel RL, Ferrante AW Jr: Obesity is associated with macrophage accumulation. J Clin Invest 2003, 112:1796-1808.

55. Okayasu M, Nakayachi M, Hayashida C, Ito J, Kaneda T, Masuhara M, Suda N, Sato T, Hakeda Y: Low-density lipoprotein receptor deficiency causes impaired osteoclastogenesis and increased bone mass in mice because of defect in osteoclastic cell-cell fusion. J Biol Chem 2012, 287:19229-19241.

56. Maeda K, Takahashi N, Kobayashi Y: Roles of Wnt signals in bone resorption during physiological and pathological states. $J$ Mol Med (Berl) 2013, 91:15-23.

doi:10.1186/ar4261

Cite this article as: Prieto-Potín et al:: Hypercholesterolemia boosts joint destruction in chronic arthritis. An experimental model aggravated by foam macrophage infiltration. Arthritis Research \& Therapy 2013 15:R81.

\section{Submit your next manuscript to BioMed Central and take full advantage of:}

- Convenient online submission

- Thorough peer review

- No space constraints or color figure charges

- Immediate publication on acceptance

- Inclusion in PubMed, CAS, Scopus and Google Scholar

- Research which is freely available for redistribution 